\title{
Stability of low back pain reporting over 8 years in a general population aged 40/41 years at base-line: data from three consecutive cross-sectional
}

\section{surveys}

Nadège Lemeunier ${ }^{1,2^{*}}$, Charlotte Leboeuf-Yde ${ }^{1,4}$, Per Kjaer ${ }^{4,5}$ and Olivier Gagey ${ }^{1,3}$

\begin{abstract}
Background: A recent review on the natural course of low back pain (LBP) in the general population indicated that the LBP reporting pattern is fairly constant over time. Furthermore, the LBP status at baseline (yes/no) seems to be predictive of the future course. When fluctuations occur, they seem most common between the nearest categories. However, in the majority of articles, non-responders were not taken into account in the analyses or interpretation of data, meaning that estimates may have been biased. Further, all reviewed studies included study participants of many different age groups. Data from three cross-sectional surveys over 8 years of the same cohort made it possible to answer the following questions: 1) Would the prevalence estimates of LBP be stable over time? 2) How would results change when taking into account non-responders? 3) Is the LBP reporting over the three survey periods stable at an individual level, taking into account also the non-responding group?

Methods: Data from three subsequent cross-sectional surveys of a study sample were available and questions about LBP were asked at baseline and also 4 and 8 years later. Study participants were 40/41 years at base-line and initially randomly selected from the general Danish population. Data were analyzed with STATA/IC 12, and presented with percentages and 95\% confidence intervals.

Results: The majority of participants reported to have had LBP in the preceding year but not having taken sick leave in relation to this pain. LBP was stable or relatively stable for the study participants as they progressed through their fifth decade. This was true on a population basis and also on an individual level. When nonresponders were taken into account the results did not change.
\end{abstract}

Conclusions: This study confirmed the results from our recent review; both presence and absence of LBP seem to be predictive for the future course. The percentage of non-responders in this type of study may not be as important as previously thought in relation to the presence/absence of LBP.

Keywords: Epidemiology, General population, Cohort, Cross-sectional study, Prevalence, Low back pain, Trajectory, Non-responders

\footnotetext{
* Correspondence: nlemeunier@ifec.net

'Complexité, Innovation et Activités Motrices et Sportives, Bâtiment 335, UFR

STAPS, Université d'Orsay Paris Sud 11, Orsay Cédex 91405, France

${ }^{2}$ Institut Franco-Européen de Chiropraxie, 72 Chemin de la Flambère,

Toulouse 31300, France

Full list of author information is available at the end of the article
} 


\section{Background}

Low back pain (LBP) is a wide-spread condition in the general population with an annual prevalence in many studies shown to be at least $50 \%$ [1]. It is difficult to diagnose [2], and because the causes are not well understood it is also difficult to treat and to prevent.

Previously, LBP was considered a disease with spontaneous cure and it was classified according to the anatomical location and duration of the pain (i.e. acute, sub acute or chronic LBP) [3]. Nowadays, it is becoming increasingly clear that LBP is more of a recurring or chronic condition, both in clinical $[4,5]$ and non-clinical populations [6], with a fluctuating course over time [7]. However, not much is known of this fluctuating pattern.

A recent review of eight studies on the natural course of LBP in the general population indicated that the LBP reporting pattern (i.e. LBP yes/no) is fairly constant regardless whether people are surveyed at short or long intervals, many times per year or with several years' intervals and also regardless if the study population is a general population or a working population [6]. In other words, those who have LBP at the onset of the observation period are likely to report it again at subsequent surveys and those without LBP keep on reporting none. When fluctuations occur between studies, they seem most common between neighboring categories, so that those with some LBP in one survey may develop into having more or none in a subsequent survey, but rarely will those without LBP develop persistent or severe LBP nor will those with persistent of severe LBP recover so as to report none [8-11].

Although the findings in the aforementioned review were consistent, it was noted that in all studies age groups were mixed, which might hide subgroup differences in relation to age, if persistence of LBP is age-dependent. Further, in all studies but one, non-responders were not taken into account but simply removed from the analyses, or at least from the interpretation of data, meaning that estimates of LBP may have been inflated or deflated in a nontransparent manner depending on the profiles and sizes of the groups of drop-out subjects. The question of how constant LBP is in the general population is important from a public health perspective. It is therefore relevant to look into this issue in some more detail.

We had access to data on LBP from three consecutive surveys of a study sample which was 40/41-years at baseline, initially randomly selected from the general Danish population, in which questions had been asked about their LBP also 4 and 8 years later. These data made it possible to obtain answers to the following questions:

1. Would the prevalence estimates of LBP be stable over time, in study subjects aged 40/41 at base-line, and aged $44 / 45$ and $48 / 49$ at the two subsequent follow-ups?
2. How would results change when taking into account non-responders?

3. Was the LBP reporting over the three survey periods stable at an individual level, taking into account also the non-responding group?

However, because not all study subjects participated at each survey, we also considered if the three overlapping samples were similar over time in relation to gender, employment status and educational level, presence or not of LBP past year, and numbers of days with LBP and sick leave in the preceding year.

\section{Methods}

Design, brief description of the study, and ethics approval This report is based on a secondary analysis of data from three consecutive cross-sectional surveys on the same cohort, a sample from the Danish general population. The first survey was conducted in 2000/01 and the others again 4 and 8 years later.

The Danish Regional Committee of Ethics approved this project ( $\mathrm{N}^{\circ} 20000042$ and $\left.96 / 272\right)$ and the database was approved by the Danish Data Protection Agency ( $\mathrm{N}^{\circ}$ 2000-53-0037).

\section{Description of the initial study sample}

In 2001, 625 people aged 40/41, living in the county of Funen (Denmark) were randomly chosen by the Central office of Civil Registration with the aim of being representative of the general Danish population. Of these, 412 (66\%) participated in survey 1 and the response rate was 84\% (348/412 and 293/348), in both survey 2 and survey 3. Please, see flow-chart Figure 1.

The study sample at base-line was previously shown to be only slightly different from the Danish general population on the level of education and the employment status variables [12]. People with basic school and vocational education/training were slightly under-represented while the short and medium levels of education were slightly over-represented [12].

\section{Data collection}

At baseline, questions were asked on employment status and education, lifestyle and the history of LBP and sick leave. The LBP questionnaire was previously validated in the Danish population for reliability and reproducibility $[13,14]$. The questions about LBP and sick leave were identical at each survey. Participants were also given a physical examination and an MRI examination.

At the two follow-up surveys, participants filled in a questionnaire at the research site, followed by an MRI scan. In this article, data on LBP contained in the three questionnaires were used. 


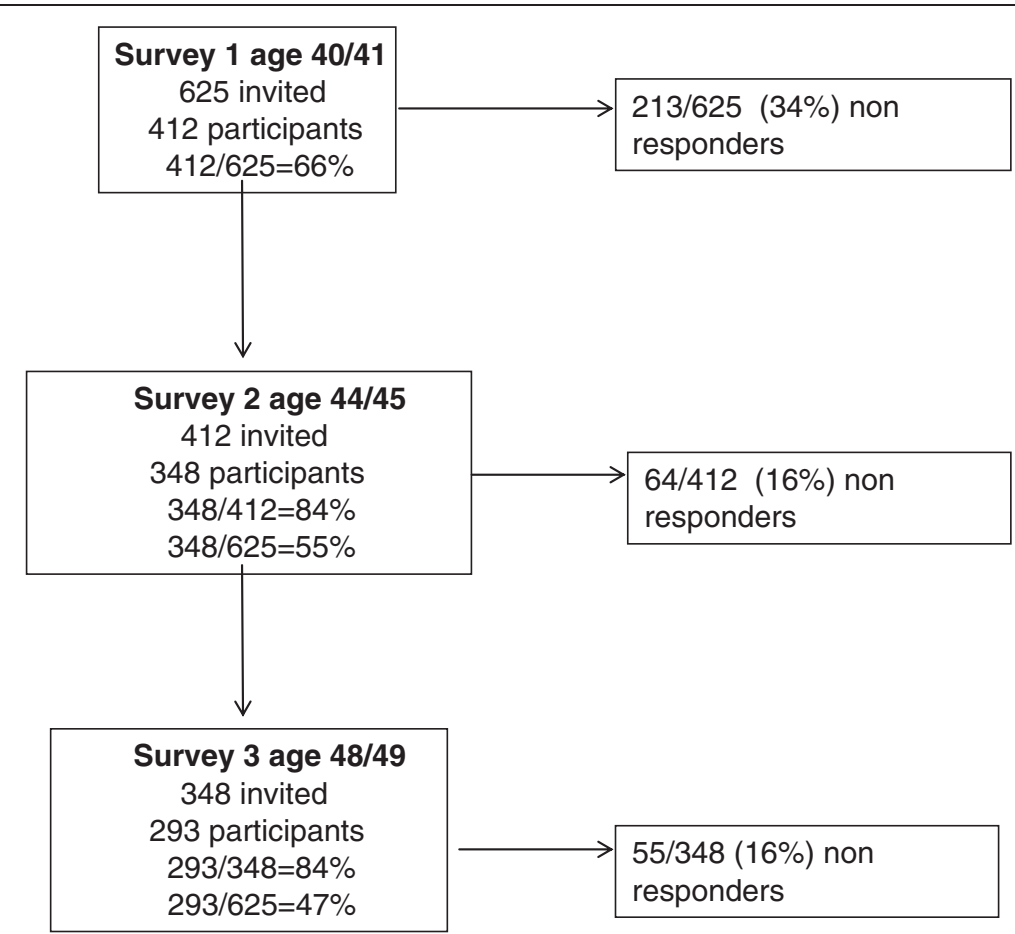

Figure 1 Flow-chart showing the study sample sizes and response rates at three subsequent surveys.

Included in the questionnaires were two questions on LBP in the preceding year ("Have you in the past year been bothered by your low back?" and "For how many days have you been bothered by your lower back within the past year?") and one on sick-leave ("For how many days have you been on sick leave because of low back trouble within the preceding year?"). The question on number of days with LBP was used as the outcome variable.

\section{Variables of interest}

From the base-line questionnaire, the following background variables were taken into account:

- Sex

- Six types of employment status: self employed, assisting spouse (i.e. assisting self-employed partner), employed, unemployed, pensioners and others outside the labor force

- Six levels of education: basic school, general uppersecondary education, vocational education/training, short-cycle higher education, medium-cycle higher education, and long-cycle higher education.

The following two variables were used from each of the three surveys:

- LBP in the past year (yes, no) and total number of days with LBP in the past year $(0,1-30,>30$ days)
- Total number of days with sick-leave because of LBP in the past year $(0,1-30,>30$ days; yes/no).

The outcome variable (LBP in the past year) was classified into $0,1-30$ and $>30$ days in accordance with previous Danish epidemiologic back pain studies [8,15-23].

\section{Data management and analysis}

The analysis of data was divided into three stages: 1) Description of the study samples, 2) Representativeness at the subsequent surveys, and 3) Research questions.

1) A description of the study sample at base-line was made with the background, LBP and sick-leave variables. Further, the number of days with sickleave in the past year in relation to the number of days with LBP in the preceding 12 months was cross-tabulated at each survey, in order to further describe the study sample.

2) Comparisons were made for the same descriptive variables between responders and non-responders at the second and the third surveys.

3) To obtain answers to the research questions, the non-responders at survey 2 and survey 3 had to be taken into account.

a. The LBP prevalence estimates were therefore calculated at each survey using three different methods. 
i. Prevalence estimates of LBP in surveys are calculated on the basis of the people participating in that survey. However, in follow-up surveys, there is often a considerable group of non-responders, who are usually ignored [6]. We called this the "usual" method and calculated the prevalence estimate at the second and third surveys based on the number of participants at that respective survey, i.e. $x$ persons with LBP divided by all the participants at the relevant survey (multiplied by 100$)$.

ii. The second method was based on the assumption that LBP does not change much over time [6]. To calculate the prevalence estimates in surveys 2 and 3, we therefore used the "same as before" method. When calculating the prevalence estimates, the nonresponders in a given survey were therefore allocated to the same category as where they were found in the preceding survey.

iii. The third method was based on the assumption that the non-responders were likely to have moved into the worst category (>30 days). The reason for this assumption was that we considered it possible that the most disadvantaged and ill were most likely to become non-responders in a survey of this type. When calculating the prevalence estimates in surveys 2 and 3, non-responders were therefore allocated to the category of $>30$ days.

b. To study the individual course of days with LBP over the period of the three surveys, cross tabulations were made for survey 1 vs. survey 2 , and survey 2 vs. survey 3 , showing also the relative size of the non-responders. The purpose of these analyses was to identify the most common patterns of change over time. This transition was described as "stayed in same category" and "moved to another category". If there was a move to another category, it was described to which one.

All data were analyzed with STATA/IC 12. Percentages and $95 \%$ confidence intervals $[\mathrm{CI}]$ were provided. Percentages were rounded up to the nearest whole figure, hence percentages do not always add up to 100 .

\section{Results}

\section{Description of study sample}

As this is a secondary analysis, information on sample sizes and response rates were given in the Methods section but is provided also in Figure 1. The majority of participants were female (52, 54 and 54\% at each survey, respectively). LBP in the preceding year was reported by almost $70 \%$ at the three surveys. In all three surveys, sick-leave had not been taken in the preceding year in the majority of cases (approximately 80\%) (Table 1). Over the three surveys, of those with 0 days of LBP, 1-2\% reported to have taken sick-leave because of LBP in the preceding year. Among those with 1-30 days of LBP past year, 15\% $18 \%$ had taken some sick-leave, usually for 1-30 days, whereas $29 \%-47 \%$ of those with LBP $>30$ days had taken some sick-leave, mainly either $1-30$ days or $>30$ days (detailed data available from the authors on request).

\section{Comparison responders/non responders}

A comparison of the final study sample in the first survey and the target population was reported in the Methods section. As seen in Table 1, there were also no big differences between responders and non-responders at the second and third surveys. However, employment status and educational level profiles became somewhat different at the second and third surveys as compared to the first. Those with LBP, particularly those with more than 30 days, and those who reported to have had LBP related sick-leave tended to become non-responders more frequently than the others.

\section{The one year period prevalence estimates}

With the "usual method" of calculating prevalence estimates, these were found to be stable over time. LBP for 1 to 30 days was most commonly reported whereas LBP $>30$ days was found in about one quarter, at all three surveys (Table 2, columns 2, 3 and 6).

If the non-responders would carry forward their "preceding" LBP status or if they would move into the "worst" category (>30 days), no changes would occur to the prevalence rates calculated the first time (Table 2, columns 4 and 7; and columns 5 and 8 , respectively).

However, the true prevalence of LBP in this population is not known because $34 \%$ of the target sample failed to participate in the study at base-line and the percentages of non-responders of the remaining sample in the second and third surveys were $15 \%$ and $29 \%$, respectively, when calculated from the base-line survey sample.

\section{Individual course}

Cross tabulations of the LBP variables between the surveys showed the individual transition of LBP over the study period for survey 1 vs. survey 2 (Table 3 ) and survey 2 vs. survey 3 (Table 4).

For both tables, the most common finding in each row is to remain in the same category over time. The second most common finding is to move up or down to one of the neighboring categories. For those with more than 
Table 1 Comparison of social factors, low back pain (LBP) and sickleave obtained at baseline for responders and non-responders at three subsequent surveys (Survey 1 , survey 2 and survey 3 )

\begin{tabular}{|c|c|c|c|c|c|c|}
\hline \multirow[t]{2}{*}{ Variables of interest } & \multicolumn{2}{|c|}{ Survey 1} & \multicolumn{2}{|c|}{ Survey 2} & \multicolumn{2}{|c|}{ Survey 3} \\
\hline & $\begin{array}{c}\text { Responders } \\
\%[\mathrm{Cl}](\mathrm{n}=\mathbf{4 1 2})\end{array}$ & $\begin{array}{c}\text { Non responders } \\
\%[\mathrm{Cl}](\mathrm{n}=\mathbf{2 1 3})\end{array}$ & $\begin{array}{c}\text { Responders } \\
\%[\mathrm{Cl}](\mathrm{n}=348)\end{array}$ & $\begin{array}{c}\text { Non responders } \\
\%[C I](n=64)\end{array}$ & $\begin{array}{c}\text { Responders } \\
\%[\mathrm{Cl}](n=293)\end{array}$ & $\begin{array}{l}\text { Non responders } \\
\%[\mathrm{Cl}](\mathrm{n}=119)\end{array}$ \\
\hline \multicolumn{7}{|l|}{ Gender } \\
\hline Female & $52[47-57]$ & $45[38-52]$ & 54 [49-59] & $41[29-53]$ & $54[48-60]$ & $47[38-56]$ \\
\hline \multicolumn{7}{|l|}{ Employment status } \\
\hline Self employed & $7[5-9]$ & - & $7[4-10]$ & $8[1-15]$ & $7[4-10]$ & $8[3-13]$ \\
\hline Assisting spouse & 0 & - & $1[0-2]$ & 0 & 0 & $1[0-3]$ \\
\hline Employed & 84 [80-88] & - & 86 [82-90] & $72[61-83]$ & 87 [83-91] & $76[68-84]$ \\
\hline Unemployed & $4[2-6]$ & - & $3[1-5]$ & $9[2-16]$ & $3[1-5]$ & 8 [3-13] \\
\hline Pensioner & $3[1-5]$ & - & $2[1-3]$ & $8[1-15]$ & $1[0-2]$ & $6[2-10]$ \\
\hline Others outside labor force & $2[1-3]$ & - & $2[1-3]$ & $3[0-7]$ & $2[0-4]$ & $3[0-6]$ \\
\hline \multicolumn{7}{|l|}{ Highest educational level } \\
\hline Basic school & 22 [18-26] & - & $21[17-25]$ & 28 [17-39] & 19 [15-23] & 30 [22-38] \\
\hline General upper-secondary education & $2[1-3]$ & - & $3[1-5]$ & 0 & $2[0-4]$ & $3[0-6]$ \\
\hline Vocational education/training & $31[27-35]$ & - & $32[27-37]$ & $25[14-36]$ & 34 [29-39] & $24[16-32]$ \\
\hline Short-cycle higher education & 20 [16-24] & - & $21[17-25]$ & $17[8-26]$ & $21[16-26]$ & 19 [12-26] \\
\hline Medium-cycle higher education & 19 [15-23] & - & 18 [14-22] & 23 [13-33] & 19 [15-23] & 18 [11-25] \\
\hline Long-cycle higher education & $6[4-8]$ & - & $5[3-7]$ & $6[0-12]$ & $5[3-7]$ & $7[2-12]$ \\
\hline \multicolumn{7}{|l|}{ LBP past year } \\
\hline Yes & $69[65-73]$ & - & 68 [63-73] & $77[67-87]$ & $69[64-74]$ & $76[68-84]$ \\
\hline \multicolumn{7}{|l|}{ Number of days with LBP past year } \\
\hline 0 & $30[26-34]$ & - & $32[27-37]$ & 20 [10-30] & $32[27-37]$ & 24 [16-32] \\
\hline $1-30$ & $45[40-50]$ & - & $45[40-50]$ & $47[35-59]$ & $46[40-52]$ & $43[34-52]$ \\
\hline$>30$ & 25 [21-29] & - & 23 [19-27] & $33[21-45]$ & $21[19-24]$ & $34[25-43]$ \\
\hline \multicolumn{7}{|l|}{ Sick-leave past year because of LBP } \\
\hline No & $80[76-84]$ & - & $84[80-88]$ & 80 [70-90] & 85 [81-89] & $74[66-82]$ \\
\hline \multicolumn{7}{|c|}{ Number of days with sick-leave past year } \\
\hline 0 & $80[76-84]$ & - & $80[76-84]$ & $80[70-90]$ & $82[78-86]$ & $74[66-82]$ \\
\hline $1-30$ & 15 [12-18] & - & 15 [11-19] & $13[5-21]$ & $13[9-17]$ & $18[11-25]$ \\
\hline$>30$ & $5[3-7]$ & - & $5[3-7]$ & 8 [1-15] & $4[2-6]$ & 8 [3-13] \\
\hline
\end{tabular}

Values among non-responders at survey 2 and survey 3 that deviate the most from the baseline estimates of the responders are identified in bold.

30 days, though, at the second survey, the most common move was to exit the study.

\section{Discussion}

To our knowledge, this is the first epidemiologic followup study in the general population, in which the transition of LBP was investigated for individuals born the same year. Identical LBP questions were asked three times in a row over a period of eight years and an identical definition of LBP was provided for the participants at each survey. The outcome variable has been extensively used and validated previously.

The results confirm what others have found [6], namely that the self-reported duration of LBP is fairly stable on a population basis. According to the past literature, this seems to be the case regardless how LBP is defined and how often and at what interval it is surveyed [6]. In our case, LBP was categorized in relation to its total duration in the preceding year; $0,1-30$, and $>30$ days.

Further, LBP fluctuates between surveys, usually by moving only one category up or down but not from extreme to extreme, again, confirming results indicated in previous research [6] that LBP is a stable condition also on an individual level. In particular, two similar studies to ours were identified $[8,9]$, both performing three surveys over a period of 5 and 9 years, respectively but on people of varying age. Their outcome variable was also 
Table 2 Days with low back pain (LBP) in the preceding year in a Danish general population aged 40/41 at baseline surveyed 3 times over 8 years

\begin{tabular}{|c|c|c|c|c|c|c|c|}
\hline & \multirow{2}{*}{$\begin{array}{c}\text { Survey } 1 \\
\text { Including the } \\
\text { responders only }\end{array}$} & \multicolumn{3}{|c|}{ Survey 2} & \multicolumn{3}{|c|}{ Survey 3} \\
\hline & & $\begin{array}{l}\text { Including the } \\
\text { responders } \\
\text { only }\end{array}$ & $\begin{array}{l}\text { SAME AS BEFORE SCENARIO: } \\
\text { Including the non } \\
\text { responders categorized } \\
\text { under their previous } \\
\text { sub-group }\end{array}$ & $\begin{array}{l}\text { WORST CASE SCENARIO: } \\
\text { Including the non } \\
\text { responders categorized } \\
\text { under the worst } \\
\text { sub-group }(>30)\end{array}$ & $\begin{array}{l}\text { Including the } \\
\text { responders only }\end{array}$ & $\begin{array}{l}\text { SAME AS BEFORE SCENARIO: } \\
\text { Including the non } \\
\text { responders categorized } \\
\text { under their previous } \\
\text { sub-group }\end{array}$ & $\begin{array}{l}\text { WORST CASE SCENARIO: } \\
\text { Including the non } \\
\text { responders categorized } \\
\text { under the worst } \\
\text { sub-group }(>30)\end{array}$ \\
\hline & $N=412$ & $N=348$ & $\mathrm{~N}=412$ & $\mathrm{~N}=412$ & $N=293$ & $\mathrm{~N}=348$ & $\mathrm{~N}=348$ \\
\hline $\begin{array}{l}\text { Number of days } \\
\text { with LBP past year }\end{array}$ & $\%[95 \% \mathrm{Cl}]$ & $\%[95 \% \mathrm{Cl}]$ & $\%[95 \% \mathrm{Cl}]$ & $\%[95 \% \mathrm{Cl}]$ & $\%[95 \% \mathrm{Cl}]$ & $\%[95 \% \mathrm{Cl}]$ & $\%[95 \% \mathrm{Cl}]$ \\
\hline 0 & $30[26-34]$ & $32[27-37]$ & $30[26-34]$ & $27[23-31]$ & $25[20-30]$ & $25[20-30]$ & $21[17-25]$ \\
\hline $1-30$ & $45[40-50]$ & $42[37-47]$ & $43[38-48]$ & $35[30-40]$ & $52[46-58]$ & $50[45-55]$ & 44 [39-49] \\
\hline$>30$ & $25[21-29]$ & $26[21-31]$ & $27[23-31]$ & 37 [32-42] & 24 [19-29] & 25 [20-30] & $36[31-41]$ \\
\hline
\end{tabular}

Prevalence estimates have been calculated in 3 different ways: including 1) the responders only; 2) the non responders categorized under their previous sub-group; and 3) the non responders categorized under the worst sub-group ( $>30$ days). 
Table 3 LBP in a Danish general population at ages 40/41 (survey 1) and at 44/45 (survey 2)

\begin{tabular}{|c|c|c|c|c|}
\hline \multirow{3}{*}{$\begin{array}{l}\text { Number of days with LBP } \\
\text { in the preceding year at } \\
\text { survey } 1(\mathrm{~N}=412)\end{array}$} & \multicolumn{4}{|c|}{$\begin{array}{l}\text { Number of days with LBP in the preceding year at survey } 2 \text { in relation to survey } 1(\mathrm{~N}=348 \text { participants }+64 \\
\text { non-responders) } \%[95 \% \mathrm{Cl}]\end{array}$} \\
\hline & 0 & $1-30$ & $>30$ & Non-responders \\
\hline & $(n=112)$ & $(n=146)$ & $(n=90)$ & $(n=64)$ \\
\hline $\mathbf{0}(\mathrm{n}=123)$ & 44 [35-53] & 38 [29-47] & 7 [2-12] & $11[5-17]$ \\
\hline $\mathbf{1 - 3 0}(\mathrm{n}=187)$ & 24 [18-30] & $44[37-51]$ & 16 [11-21] & 16 [11-21] \\
\hline$>\mathbf{3 0}(\mathrm{n}=102)$ & 14 [7-21] & 16 [9-23] & 50 [40-60] & 21 [13-29] \\
\hline
\end{tabular}

Cross tabulation of the number of days with LBP in the past year for survey 1 vs. survey 2 when taking non-responders into account. The table shows the proportions of people in each category in percentages (\%) with $95 \%$ confidence intervals $(95 \% \mathrm{Cl})$ which were found in the same or in a different category of LBP duration at the 2 nd survey as compared to the 1 st survey.

number of days with LBP in the preceding year. Their data included information on stability of both absence and presence of LBP or fluctuations between "neighboring" categories, as in our own study. Their results concur with ours.

In epidemiologic research, sampling method, sample size and response rates are important issues, as they may have an influence on the representativeness of the final study sample and hence on the external validity of the results. Unfortunately, it is difficult to motivate people from the general population to participate in studies, making high response rates hard to achieve, in particular over a series of surveys over a prolonged period of time carried out on the same cohort. For example, according to our previous review that included epidemiologic studies of the general population, the real response rates at the last follow-up ranged between $21 \%$ and $76 \%$, when taking into account the participants at the first survey [6]. The consequences of this can be difficult to ascertain and are only rarely addressed in studies similar to this one.

Our response rates seemed high, when calculated as it is usually done, based on the number of people participating at each survey in relation to the participants at the previous survey. Nevertheless, these percentages would diminish, as is usually the case also in other studies, if the entire target sample were to be taken into account when calculating response rates. We therefore looked closer at the proportion of non-responders throughout our three surveys and noted that non- response was particularly common at base-line (approximately $1 / 3$ of those invited were missing), meaning that already the initial study sample may be biased in some (unknown) way. At survey 2, 1/6 of the remaining sample disappeared and then again at survey 3 an additional $1 / 6$, resulting in, at survey 3 , a remaining group consisting of approximately half of those invited at the very beginning. This resulted in some differences between responders and non-responders, providing a hint on how to interpret the final data. We therefore found it relevant to demonstrate the size of the non-responder groups in our result tables.

In relation to the LBP variable, differences between responders and non-responders at the two follow-ups were noticed with relatively more losses to follow-up among those with LBP $>30$ days than in the other two categories. Interestingly, though, the proportions of people who dropped out of our study were similar for each base-line category of LBP $(11 \%-21 \%$ and $14 \%-21 \%$ at surveys 2 and 3 , respectively). As, on an individual level, the stability of LBP reporting over time was high, the estimates would therefore not change much over time, assuming as we did that non-responders would remain where they were at the previous survey. Thus, the stable nature of LBP may make response rates less important than generally presumed, provided that the proportion of dropouts is evenly distributed among the initial subgroups. However, this would be better understood with more detailed information on the nature of LBP in relation to severity and consequences.

Table 4 LBP in a Danish general population at ages 44/45 (survey 2) and at 48/49 (survey 3 )

\begin{tabular}{|c|c|c|c|c|}
\hline \multirow{3}{*}{$\begin{array}{l}\text { Number of days with LBP } \\
\text { in the preceding year at } \\
\text { survey } 2(n=348)\end{array}$} & \multicolumn{4}{|c|}{$\begin{array}{c}\text { Number of days with LBP in the preceding year at survey } 3 \text { in relation to survey } 2 \text { ( } N=293 \text { participants }+119 \\
\text { non-responders) } \%[95 \% \mathrm{Cl}]\end{array}$} \\
\hline & 0 & $1-30$ & $>30$ & Non-responders \\
\hline & $(n=72)$ & $(n=152)$ & $(n=69)$ & $(n=55)$ \\
\hline $\mathbf{0}(n=112)$ & 38 [29-47] & $35[26-44]$ & 8 [3-13] & 20 [13-17] \\
\hline $\mathbf{1 - 3 0}(n=146)$ & 16 [10-22] & $58[50-66]$ & 16 [10-22] & $14[8-20]$ \\
\hline$>\mathbf{3 0}(\mathrm{n}=90)$ & $8[2-14]$ & $31[21-41]$ & $40[30-50]$ & 19 [13-29] \\
\hline
\end{tabular}

Cross tabulations of the number of days with LBP in the past year for survey 2 vs. survey 3 when taking non responders into account. The table shows the proportions of people in each category in percentages (\%) with $95 \%$ confidence intervals (95\% Cl) which were found in the same or in a different category of LBP duration at the 3 rd survey as compared to the 2 nd survey. 
From a research perspective, it would be relevant to find out if this constancy is stable also over shorter periods and if it covers different subgroups with different courses on a more short-term basis. Further, it would be necessary to investigate if summary subgroups, such as ours ( 0 days, $1-30$ days and $>30$ days) really exist. How easily can one recall if the pain lasted 30 or 31 days? Can those with only a few days of pain really remember this or are they more likely to think they had had no LBP at all? Frequent data collection, such as using text messages or internet diaries, would be relevant, finally, to be able to validate this subgroup classification. Our next article will cover this issue.

\section{Conclusions}

In conclusion, LBP is a stable or relatively stable condition for individuals in the general Danish population as they progress through their fifth decade. For patients and clinicians, this is important information as it indicates that presence of LBP, once there, is a more or less normal state and that absence of LBP probably indicates a degree of "immunity" to this condition. This statement is supported, not only by our recent review of studies from the general population [6] but also by a recent review of the one-year clinical course of non-specific LBP, which concluded that non-recovery is more common than recovery [24]. It is interesting that this phenomenon is so similar both in clinical and non-clinical populations, perhaps indicating that the clinical and natural courses are more or less identical.

\section{Competing interest}

The authors declare that they have no competing interest.

\section{Authors' contributions}

PK was responsible for the epidemiologic study that formed the basis for this work. CLY, OG and NL formulated the research questions. PK and NL performed the analysis. All the authors were involved in interpreting the data, writing the manuscript and approving the final version.

\section{Acknowledgements}

The authors wish to thank Professor Tom Bendix for his role in designing the original study and securing primary funding. Furthermore, we would like to thank Professor Claus Manniche, at the Spine Centre, Ringe, Denmark for hosting the entire project. Professor Charlotte Leboeuf-Yde was partially funded, until December 31st, 2012, by Fonden til Fremme for Kiropraktisk Forskning og Postgraduat Uddannelse.

\section{Funding}

The initial project was possible based on a large donation from Danish Industrial Insurance Company, now TopDanmark.

\section{Author details}

'Complexité, Innovation et Activités Motrices et Sportives, Bâtiment 335, UFR STAPS, Université d'Orsay Paris Sud 11, Orsay Cédex 91405, France. ${ }^{2}$ Institut Franco-Européen de Chiropraxie, 72 Chemin de la Flambère, Toulouse 31300, France. ${ }^{3}$ Bicêtre University Hospital, AH-HP Paris, F-94270, JE-2494 Université paris-Sud, Orsay F-91405, France. ${ }^{4}$ Research Department, Spine Center of Southern Denmark, Hospital Lillebaelt and Institute of Regional Health Services Research, Clinical Locomotion Network, University of Southern Denmark, Ostre Hougvej 55, Middelfart, Denmark. ${ }^{5}$ Institute of Sports Science and Clinical Biomechanics, Clinical Locomotion Network, University of Southern Denmark, Odense, Denmark.

Received: 21 June 2013 Accepted: 13 September 2013

Published: 21 September 2013

\section{References}

1. Lebœuf-Yde C, Fejer R, Nielsen J, OKyvik K, Hartvigsen J: Consequences of spinal pain: Do age and gender matter? A cross-sectional populationbased study of 34902 individuals 20-71 years of age. BMC Musculoskelet Disord 2011, 12:39.

2. Deyo R, Rainville J, Kent D: What can the history and physical examination tell us about low back pain? JAMA 1992, 6:760.

3. Spitzer WO, LeBlanc RE, Dupuis MS: Scientific approach to the assessment and management of activity-related spinal disorders. In Monograph for Clinicians: Report of the Quebec Task Force on Spinal Disorders. Spine 1987, 12:s16-s21.

4. van den Hoogen HJ, Koes BW, van Eijk JT, Bouter LM, Deville W: On the course of low back pain in general practice: a one year follow up study. Ann Rheum Dis 1998, 57(1):13-19.

5. Stanton TRL, Maher CG, Hancock MJ: How do we define the condition 'recurrent low back pain'? A systematic review. Eur Spine J 2010, 19:533-539.

6. Lemeunier N, Lebœuf-Yde C, Gagey O: The natural course of low back pain: a systematic critical literature review. Chiropr Man Therap 2012, 20(1):33.

7. Tamcan O, Mannion AF, Eisenring C, Horisberger B, Elfering A, Müller U: The course of chronic and recurrent low back pain in the general population. Pain 2010, 150(3):451-457.

8. Hestbæk L, Lebœuf-Yde C, Engberg M, Lauritzen T, Bruun NH, Manniche C: The course of low back pain in a general population. Results from a 5-year prospective study. J Manipulative Physiol Ther 2003, 26(4):213-219.

9. Maul I, Läubli T, Klipstein A, Krueger H: Course of low back pain among nurses: a longitudinal study across eight years. Occup Environ Med 2003, 60:497-503

10. van Oostrom SH, Verschuren VMM, de Vet HCW, Picavet HS: Ten year course of low back pain in an adult population-based cohort - The Doetinchem Cohort Study. Eur J Pain 2011, 15:993-998.

11. Videman T, Ojajärvi A, Riihimäki H, Troup JDH: Low back pain among nurses. Spine 2005, 30(20):2334-2341.

12. Kjaer P, Lebœuf-Yde C, Korsholm L, Sorensen JS, Bendix T: Magnetic resonance imaging and low back pain in adults: a diagnostic imaging study of 40-year-old men and women. Spine 2005, 30(10):1173-1180.

13. Biering-Sørensen F: A one year prospective study of low back trouble in a general population. The prognostic value of low back history and physical measurement. Dan Med Bull 1984, 31:362-375.

14. Kuorinka I, Jonsson B, Kilbom A, Vinterberg H, Biering-Sørensen F, Andersson G, Jørgensen K: Standardized Nordic questionnaire for the analysis of musculoskeletal symptoms. Appl Ergon 1987, 18:233-237.

15. Lebœuf-Yde C, Yashin A, Lauritzen T: Does smoking cause low back pain? Results from a population-based study. J Manipulative Physiol Ther 1996, 19:99-108.

16. Lebœuf-Yde C, Klougart N, Lauritzen T: How common is low back pain in the Nordic population? Data from a recent study on a middle-aged general Danish population and four surveys previously conducted in the Nordic countries. Spine 1996, 21:1518-1526

17. Lebœuf-Yde C, Lauritsen J, Lauritzen T: Why has the search for causes of low back pain largely been nonconclusive. Spine 1997, 22:877-881.

18. Lebœuf-Yde C, Ohm Kyvik K: At what age does low back pain become a common problem? A study of 29,424 individuals aged 12-41 years. Spine 1998, 23:228-234.

19. Lebœuf-Yde C, Ohm Kyvik K, Bruun H: Low back pain and life-style. Part I: Smoking. Information from a population-based sample of 29,424 twins. Spine 1998, 23:2207-2214.

20. Lebøuf-Yde C, Ohm Kyvik K, Bruun H: Low back pain and life-style. Part II Obesity. Information from a population-based sample of 29,424 twin subjects. Spine 1999, 24:779-784.

21. Hartvigsen J, Bakketeig LS, Lebœuf-Yde C, Engberg M, Lauritzen T: The association between physical workload and low back pain clouded by the "healthy worker" effect. Population-based cross-sectional and 5-year prospective questionnaire study. Spine 2001, 26:1788-1793. 
22. Hestbæk L, Lebœuf-Yde C, Kyvik KO, Manniche C: Is low back pain in youth associated with weight at birth? A cohort study of 8,000 Danish adolescents. Dan Med Bull 2003, 50:181-185.

23. Hartvigsen J, Kyvik KO, Lebœuf-Yde C, Lings S, Bakketeig L: Ambiguous relation between physical workload and low back pain: a twin control study. Occup Environ Med 2003, 60:109-114.

24. Itz CJ, Geurts JW, van Kleef M, Nelemans P: Clinical course of non-specific low back pain: a systematic review of prospective cohort studies set in care. Eur J Pain 2013, 17(1):5-15.

doi:10.1186/1471-2474-14-270

Cite this article as: Lemeunier et al: Stability of low back pain reporting

over 8 years in a general population aged 40/41 years at base-line: data

from three consecutive cross-sectional surveys. BMC Musculoskeletal Disorders 2013 14:270.

\section{Submit your next manuscript to BioMed Central and take full advantage of:}

- Convenient online submission

- Thorough peer review

- No space constraints or color figure charges

- Immediate publication on acceptance

- Inclusion in PubMed, CAS, Scopus and Google Scholar

- Research which is freely available for redistribution 